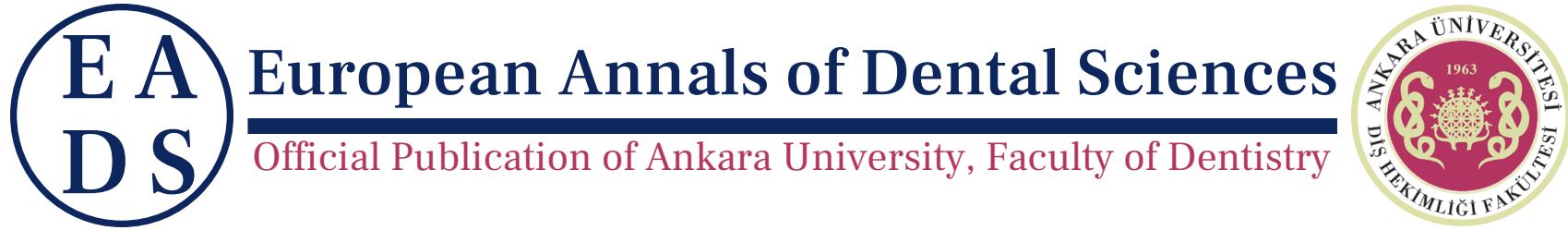

EADS, 2021, 48 (2), 74-77

e-ISSN : 2757-6744

Article Received : February, 232021

doi : 10.52037/eads.2021.0016

Article Accepted : May, 302021

\title{
Brown tumor of the mandible as an initial presentation of hyperparathyroidism
}

\author{
Antigone Delantoni ${ }^{1}$, Merve Önder $\odot 2, *$ and Kaan Orhan $\odot 3$
}

${ }^{1} \mathrm{DDS}$, PhD, Dept of Oral Surgery, Implant Surgery and Radiology, Faculty of Dentistry, Aristotle University of Thessaloniki, Greece and ${ }^{2}$ DDS, Ankara University, Faculty of Dentistry, Department of DentoMaxillofacial Radiology, Ankara, Turkey and ${ }^{3} \mathrm{DDS}$, PhD, Ankara University, Faculty of Dentistry, Department of DentoMaxillofacial Radiology, Ankara, Turkey

*Corresponding Author; merveonder_16@hotmail.com

\begin{abstract}
The paper presents a brown tumor case related to secondary hyperparathyroidism in an end stage kidney disease patient undergoing dialysis treatment. The interesting feature of the case is that the primary clinical presentation of the condition was a mild swelling in the attached gingiva of a mandibular molar tooth. Medical practitioners should be alert to the fact that some pathological conditions may have an initial presentation in the oral cavity. Thus, a thorough and careful examination of the oral mucosa with the accompanying dental radiographs of patients, should be noted and studied in all cases, where available.
\end{abstract}

Key words: brown tumor; hyperparathyroidism; mandible

\section{Introduction}

Parathyroid hormone is produced by the parathyroid glands and has a key role in bone metabolism. These glands, located behind the thyroid at the bottom of the neck, are about the size of a grain of rice. Hyperparathyroidism occur when they create too much parathyroid hormone in the bloodstream.1 Hyperparathyroidism (HPT) may be primary, secondary, and tertiary and all types are featured with the presence of overproduction of PTH. ${ }^{1-3}$

Brown tumor (BT) or osteitis fibrosa cystica is a benign osseous lesion, present in settings of excess osteoclastic activity, which may result in any form of uncontrolled PTH hypersecretion. 4,5

Brown tumours are of fibrous tissue, and woven bone origin, but no bone matrix. The osteoclasts consume the bone formed by osteoblasts and this repetition of reparative bone deposition followed by additional resorption can expand beyond the usual shape of the bone, involving the periosteum in which case there is the present of pain in the osseous area involved.

Brown tumors may be rarely associated with ectopic parathyroid adenomas or end stage kidney condition, as is the case presented in this paper. 4,5

In developed countries with the evolution of medicine and the early diagnosis of the condition, as well as the successful treatment of HPT, they are rarely seen and only in final untreated disease stages. ${ }^{5}$

We describe a patient who presented with a manifestation of the Brown tumor to the mandible manifested by orofacial alteration as the presenting symptom of Hyperthyroidisma.

\section{Case Report}

The patient was a 58 years old female Caucasian, who came to complain about a mild swelling in the attached gingivae of a mandibular molar (Figure 1). The patient's medical history only included end-stage renal disease (ESRD). She was on hemodialysis and had no other medical conditions. Upon clinical examination, a mild, painless swelling was noted in the attached gingivae of a mandibular molar. The swelling extended to the non-attached gingivae and was not movable upon palpation. No other clinical findings were noted.

The patient was sent for a panoramic radiography (Figure 2), and the presence of a radiolucent multilocular lesion in the area was detected. The lesion was located in the posterior mandible in close relation with mandibular canal but without any interference with third molar. Trabecular structure of the mandible 


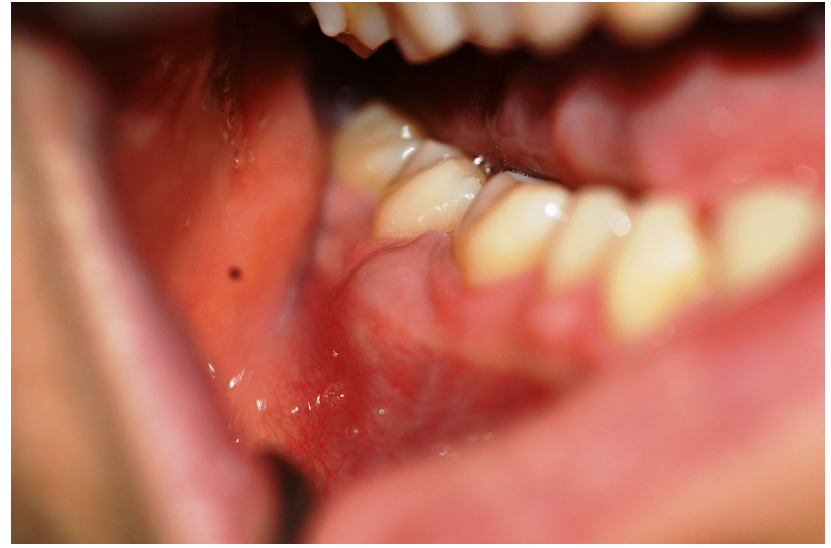

Figure 1. Clinical presentation of the patients gingival swelling

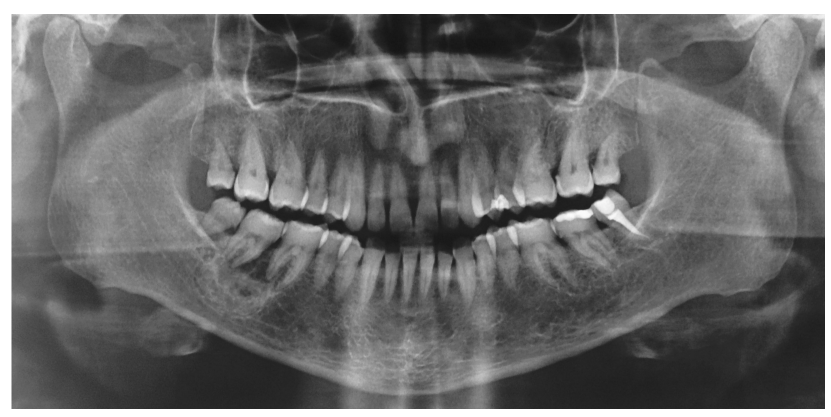

Figure 2. A: Non-specific bacterial colonies inter-mixed with inflammatory infiltrate, and aggregates of necrotic debris (10X). B: Cystic cavity lined by a non-keratinized, hyperplasic stratified squamous epithelium (10X). C: Higher power showing foci of dystrophic calcification with a basophilic appearance in variable mineralization pattern (See arrows) (20X). D: An area showing numerous curvilinear dystrophic calcification (see arrows) and aggregates of red blood cells (20X).

was altered due to do lesion there was thinning of the cortex without expansion of the mandible.

After examination with a medical CT (Figure 3), the extent of the lesion was noted. Since the patient did not have any other medical problems, a biopsy of the lesion was performed to set the diagnosis.

The histopathology of the lesion (Figure 4) showed multiple giant cells present and increased osteoclastic activity. The diagnosis of osteitis fibrosa cystica was given by the histopathology of the lesion. After the histopathology had set the diagnosis, the patient was asked to take a PTH exam and the PTH was $385 \mathrm{pg} / \mathrm{mL}$ with normal values ranging up to $55 \mathrm{pg} / \mathrm{mL}$. After the clinical, radiographic, histologic exams and the blood PTH levels, treatment of the patient was set according to the blood levels of PTH, and surgical excision of the lesion was performed.

\section{Discussion}

The parathyroid glands, situated behind the thyroid, respond to changes in serum ionized calcium concentrations and produde PTH. The hormone interacts with vitamin D and plays a key role with its metabolites in regulating calcium absorption and excretion. ${ }^{6,7}$ The increase in levels of PTH is associated with the increased osteoclastic activity, extensive bone remodeling, and osteoblastic repair.

Hyperparathyroidism is a condition that can lead to a series of complications that decrease the life quality of the patient. In patients with chronic kidney disease (CKD), the im-

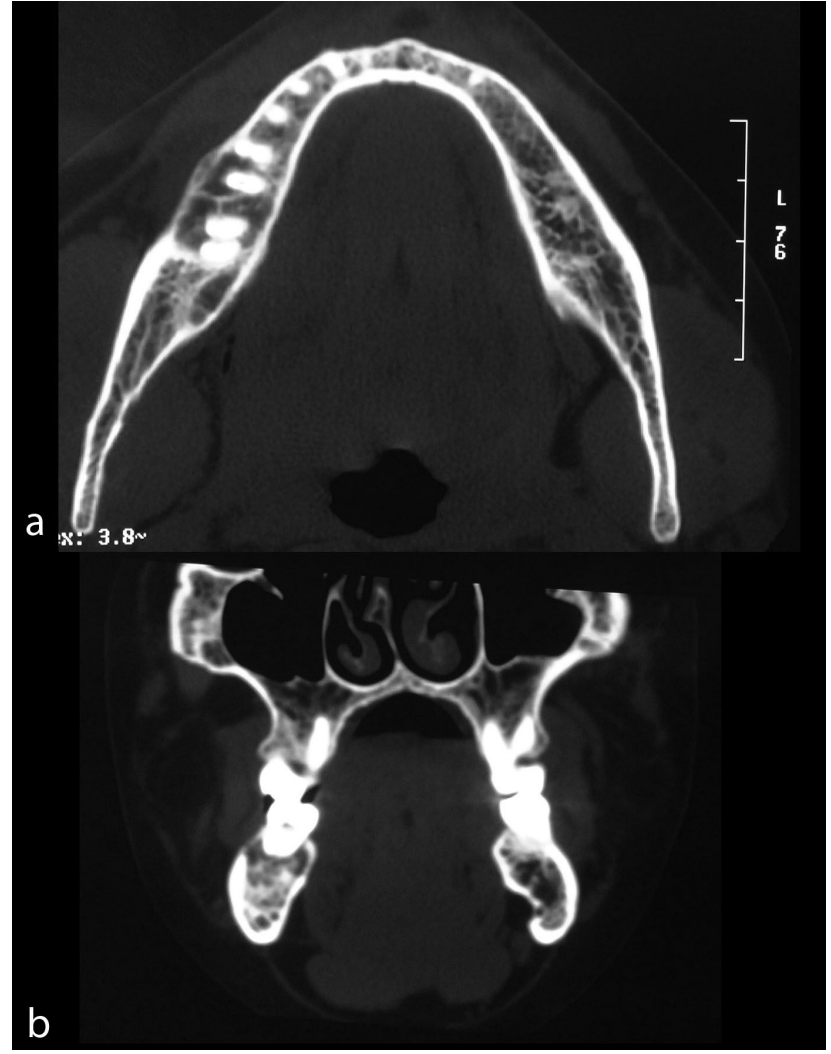

Figure 3. a: Axial b: Coronal Computer tomography of the patient showing the extend of the lesion and its limitation to bone

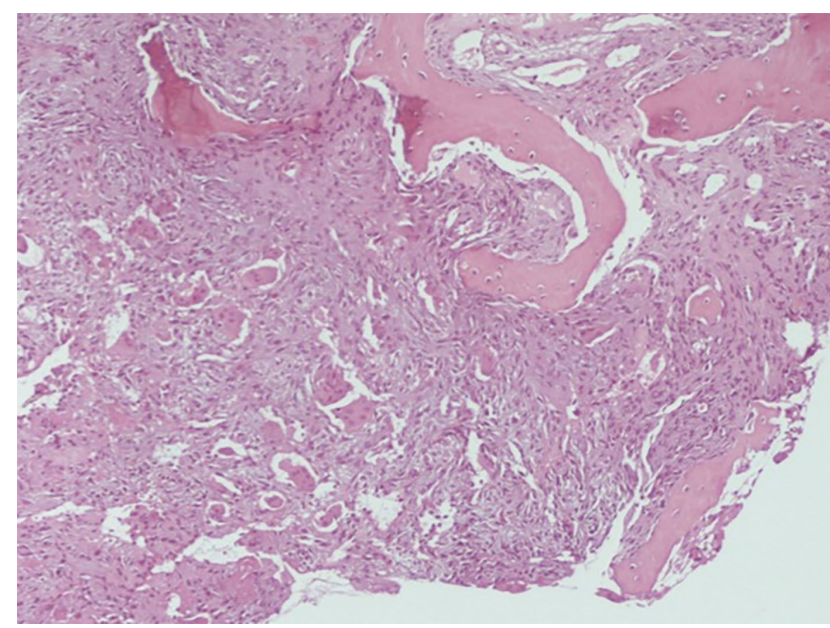

Figure 4. Histopathological section of the lesion with an increased osteoclastic activity

paired renal function leads to decreased vitamin D levels, causing an increase in parathyroid hormone (PTH) production and contributing to the development of secondary hyperparathyroidism. Lack of vitamin D leads to reduced calcium absorption by the intestine leading to hypocalcemia and increased parathyroid hormone secretion. ${ }^{8}$ The bone disease in secondary hyperparathyroidism caused by kidney failure is termed renal osteodystrophy. Tertiary hyperparathyroidism is seen in those with long-term secondary hyperparathyroidism, which eventually leads to hyperplasia of the parathyroid glands and a loss of response to serum calcium levels. This disorder is most often seen in patients with end-stage kidney disease and is an autonomous activity. ${ }^{8-10}$ 


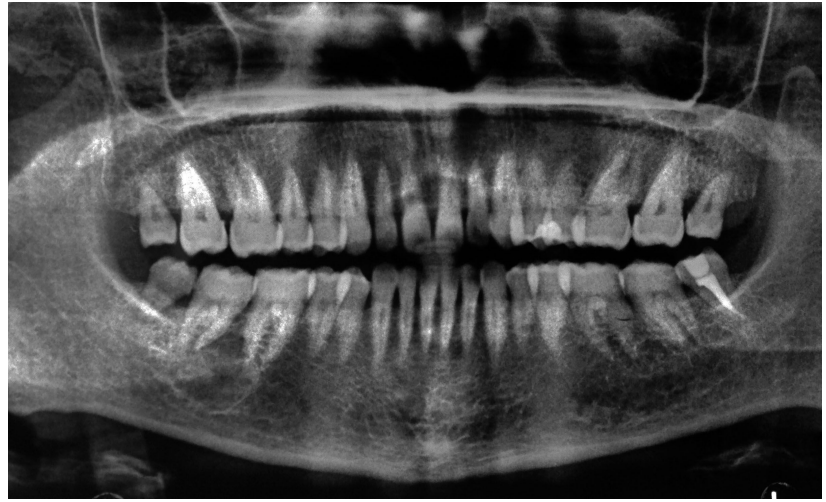

Figure 5. Histopathological section of the lesion with an increased osteoclastic activity

Diagnosis of brown tumors is merely presumptive. Histology sets the final diagnosis. Laboratory data and radiographs may be additionally used for definitive clinical diagnosis.

In radiographical examination, there are findings from the bones that can even aid the diagnosis of the condition in a large number of patients. Brown tumor may show no changes or a generalized osteoporosis image, making it difficult to diagnose. The lesions present as sharply defined, round or oval radiolucent areas which may appear multilocular. ${ }^{11}$

It is useful to know the serum levels of calcium and parathyroid hormone to set the differential diagnosis from giant cell lesions. In hyperparathyroidism as in the presented case, serum PTH level was $385 \mathrm{pg} / \mathrm{ml}$, which was indicative of the diagnosis of the brown tumor, when compared to other giant cell tumors. ${ }^{3}$

In the presented case, the patient's panoramic radiography that was taken two years ago (Figure 5), showed a very early lesion with no well-defined radiolucent osteolytic lesions near the middle section of the teeth' roots. In the present panoramic radiography of the same patient, the lesion had progressed to a well-defined lucency adjacent to the tooth's roots and extending to the surrounding bone. The lesion was removed regionally, and the systemic management revolved primarily in reducing circulating endocrine hormone (PTH). What should be noted here is that in the first panoramic radiograph though here was some indication, the diagnosis could not have been set from the radiographic image.

Similar cases have been dealt with accordingly with no need for further medical management. ${ }^{6-10}$ In late diagnoses and those that do not respond to medical treatment, parathyroidectomy is the treatment of choice.12 In our case, an open curettage biopsy was performed and sent for histopathologic evaluation. The histopathology reported it as CGCG with sections showing microcellular connective tissue stroma with fibroblasts in abundance, and multinucleated giant cells, few osteoblasts, and numerous blood vessels (Figure 5) In the present case the brown tumor of the mandible was the first sign of the condition (hyperparathyroidism) due to the inbalance of osteoblastic, osteoclastic activity, formed by increased PTH levels and calcium phosphorous serum levels regulation. ${ }^{1,9,11,12}$

Upon reexamining the patient there was no recurrence of the lesion or presence of other similar lesions. The limitation of this paper can be lack of more comprehensive imaging of the current lesion since 3D imaging with additional/surface bone reconstruction can be necessary for surgical interventions.

\section{Conclusion}

The presented case had the unusual primary detection of the condition in the mandible. This should alert radiologists, and one should be careful of the medical history of a patient even upon taking a plain radiography. The differential diagnosis should be set, only after a thorough study of the patients both medical and dental history. Dentists should be aware of the medical conditions that may cause dental problems and should be more alert to certain patients.

\section{Author Contributions}

Delantoni A examined the case, photographed and prepared. Orhan $\mathrm{K}$ investigated the case and made the necessary corrections. Onder $M$ made literature scanning and final edits.

\section{Conflict of Interest}

Authors declare that they have no conflict of interest.

\section{Authors' ORCID(s)}

$\begin{array}{ll}\text { A.D. } & 0000-0002-5679-6779 \\ \text { M.O. } & 0000-0002-3476-1727 \\ \text { K.O. } & 0000-0001-6768-0176\end{array}$

\section{References}

1. Fang Y, Hong G, Lu H, Guo Y, Yu W, Liu X. [Brown tumor: clinical, pathological and imaging manifestations]. Zhonghua Yi Xue Za Zhi. 2015;95(45):3691-3694.

2. Hu J, He S, Yang J, Ye C, Yang X, Xiao J. Management of brown tumor of spine with primary hyperparathyroidism: A case report and literature review. Medicine (Baltimore). 2019;98(14):e15007. doi:10.1097/md.0000000000015007.

3. Nassar GM, Ayus JC. Images in clinical medicine. Brown tumor in end-stage renal disease. $\mathrm{N}$ Engl J Med. 1999;341(22):1652. doi:10.1056/nejm199911253412204.

4. Keyser JS, Postma GN. Brown tumor of the mandible. Am J Otolaryngol. 1996;17(6):407-410. doi:10.1016/s01960709(96)90075-7.

5. Olsen J, Sealey C. Brown tumour of the mandible in primary hyperparathyroidism; a case report. $\mathrm{N} Z$ Z Dent J. 2015;111(3):116-118.

6. Huang R, Zhuang R, Liu Y, Li T, Huang J. Unusual presentation of primary hyperparathyroidism: report of three cases. BMC Med Imaging. 2015;15:23. doi:10.1186/s12880015-0064-1.

7. Minisola S, Gianotti L, Bhadada S, Silverberg SJ. Classical complications of primary hyperparathyroidism. Best Pract Res Clin Endocrinol Metab. 2018;32(6):791-803. doi:10.1016/j.beem.2018.09.001.

8. Shetty AD, Namitha J, James L. Brown tumor of mandible in association with primary hyperparathyroidism: a case report. J Int Oral Health. 2015;7(2):50-52.

9. Di Daniele N, Condò S, Ferrannini M, Bertoli M, Rovella $\mathrm{V}$, Di Renzo L, et al. Brown tumour in a patient with secondary hyperparathyroidism resistant to medical therapy: case report on successful treatment after subtotal parathyroidectomy. Int J Endocrinol. 2009;2009:827652. doi:10.1155/2009/827652.

10. Gulati D, Bansal V, Dubey P, Pandey S, Agrawal A. Central giant cell granuloma of posterior maxilla: first expres- 
sion of primary hyperparathyroidism. Case Rep Endocrinol. 2015;2015:170412. doi:10.1155/2015/170412.

11. Moran LM, Moeinvaziri M, Fernandez A, Sanchez R. Multiple brown tumors mistaken for bone metastases. Computed tomography imaging findings. EJRNM. 2016;47(2):537-541.
12. Reséndiz-Colosia JA, Rodríguez-Cuevas SA, Flores-Díaz R, Juan $\mathrm{MH}$, Gallegos-Hernández JF, Barroso-Bravo S, et al. Evolution of maxillofacial brown tumors after parathyroidectomy in primary hyperparathyroidism. Head Neck. 2008;30(11):1497-1504. doi:10.1002/hed.20905. 